\title{
Ultrastructural Analysis of Chromosome Translocation-Induced Neural Tube Defects
}

\author{
K. SUE O'SHEA \\ Department of Anatomy and Cell Biology, University of Michigan Medical School, Ann Arbor, MI 48109
}

\begin{abstract}
O'SHEA, K. S. Ultrastructural analysis of chromosome translocation-induced neural tube defects. BRAIN RES BULL 16(6) $833-844,1986$.- Neural tube closure defects occurred in $33 \%$ of the embryos obtained from matings of male mice heterozygous for a reciprocal chromosome translocation $(\mathrm{T}(2 ; 4) 1 \mathrm{Sn})$ with normal female CFLP mice. Light and electron microscopic observations of neuroepithelium and mesenchyme in affected embryos indicated two distinct types of anomalies occurred. The first consisted of neuroepithelial hypertrophy and neural tube closure defects. These defects most frequently affected the midbrain and hindbrain, but occasional defects of the lumbosacral neural tube were also observed. Unlike the highly organized, pseudostratified neuroepithelium in control embryos, neuroepithelial cells became stratified and formed cell islands with secondary lumina within the wall of the neural tube. The second condition was associated with a reduction in neuroepithelial thickness, considerable neuroepithelial and neural crest cell death, basal lamina alterations and premature invasion of the neuroepithelium by subjacent endothelial cells. In both cases, the cephalic mesenchyme cells, rather than their normal stellate appearance, were markedly elongated in shape and reduced in area. The number of cell-cell contacts between mesenchymal cells was also reduced significantly. These results are discussed in light of recent theories regarding the role of mesenchyme and extracellular matrix in neurulation.
\end{abstract}

Chromosome translocation Neural tube defect Neuroepithelium Mesenchyme Mouse embryo

DEFECTS of neural tube closure such as anencephaly and spina bifida are among the most severe and at the same time poorly understood conditions which may affect an infant. Little is known about the mechanisms by which they develop; both environmental and hereditary factors, as well as interactions of the two [10], have been invoked to explain their pathogenesis.

Although the majority of infants with neural tube defects (NTDs) have a normal karyotype [16, 21, 27], chromosomal abnormalities have occasionally been associated with NTDs. These include: tetraploidy [42], trisomy [9], partial duplication $(11 q)[3,59]$, balanced $(11 ; 13)$ translocation $[20,46]$, ring chromosome 13 [48], and sex chromosome abnormalities (XX male, [2]). However, only in the case of trisomy 18, in which there is a low overall incidence of major CNS defects, does it appear that NTDs may be a consistent feature of the spectrum of anomalies $[23,39,56]$.

While there are a number of genetic mutants of the mouse with dose-dependent NTDs (e.g., Splotch [47], loop-tail [54], curly-tail [11], open eyelid [57], Patch [14]), there are only a few studies in which chromosomal aneuploidy has been associated with neural tube defects. In the mouse embryo, trisomy (Ts) of chromosomes 9, 12, 14 and 17 [19] and chromosome translocation $(\mathrm{T}(2 ; 4) 1 \mathrm{Sn})[51,52]$ have been reported to produce neural tube closure defects. Interestingly, each aneuploid condition has been associated with "semispecific malformations" [19]. Among the trisomies in the mouse two produce exencephaly: trisomy 12 which affects the midbrain and hindbrain and trisomy 14 which involves the midbrain but not hindbrain [44]; while in the chromosome translocation, all areas of the neuraxis may be affected [37]. Although none of these conditions is strictly analogous to a human trisomy or translocation, there is considerable value in studying the development of neural tube defects in these animals as, unlike many teratogen-induced defects, there is a relatively high incidence of anomalies, and all stages of development are available for detailed observation of the events which produce the NTDs.

In the current investigation, the histological and ultrastructural appearance of neuroepithelium and mesenchyme was examined in control embryos and in embryos with chromosome translocation $(\mathrm{T}(2 ; 4) 1 \mathrm{Sn})$-induced neural tube defects.

\section{METHOD}

Animals

Male heterozygous translocation (T(2;4)1Sn) carriers were kindly provided by Drs. M.F. Lyon and M. K. Kirk of the MRC Radiobiology Unit (Harwell, England) and mated with 6-8 week old CFLP female mice (Anglia Labs). CFLP $\times$ CFLP matings and CD- $1 \times$ CD-1 (Charles River Labs) matings provided additional control embryos. Early on the afternoon of the 10th or 11th day of gestation (1st day $=+$ vaginal plug) females were killed by cervical dislocation, decidua removed from the uteri and embryos dissected 
into Petri dishes containing phosphate buffered saline. Embryos were dissected from decidua and chorion, examined for the presence of external anomalies, then immersion fixed for 2 hours at room temperature in a $2 \%$ solution of glutaraldehyde in $0.1 \mathrm{M}$ phosphate buffer ( $\mathrm{pH}=7.4,320 \mathrm{mOsmol}$ ). Embryos were then washed and stored in $0.1 \mathrm{M}$ phosphate buffer with sucrose to maintain the osmolarity.

For SEM examination, embryos were dehydrated through alcohols, critical point dried in $\mathrm{CO}_{2}$, sputter coated, then viewed and photographed in a Cambridge S600 or ISI DS-130 scanning electron microscope.

Embryos obtained from matings of translocation carriers with CFLP females have been designated 'Snell'; from CFLP $\times$ CFLP matings, 'CFLP control'; and from CD$1 \times$ CD -1 matings, 'CD- 1 control.'

\section{Microscopy}

For light and transmission electron microscopy, embryos were post-fixed in $1 \% \mathrm{OsO}_{4}$ for 30 minutes, dehydrated and infiltrated with Araldite resin. Thick (1-2 micron) sections were heat-affixed to glass slides and stained with toluidine blue for light microscopical observations.

Thin $(80 \mathrm{~nm})$ sections were cut using a diamond knife, double stained with lead citrate and uranyl acetate, viewed and photographed in a Philips $\mathbf{4 0 0}$ electron microscope.

\section{Morphometry}

To quantify cellular alterations observed using EM, five similar fields of mesenchymal cells from Snell $(n=5)$ and CD-1 control $(n=5)$ embryos were photographed at $1650 \times$. Only cells cut so that the nucleus was included in the plane of section were analyzed; portions of cells and cell processes were therefore not included. In addition, care was taken to sample mesenchyme between (not touching) neuroepithelium and lateral surface ectoderm. A total of 188 Snell and $153 \mathrm{CD}-1$ control mesenchymal cells were measured. Prints were made and cell areas, perimeters, greatest lengths and number of cell-cell contacts were measured using a digitizing pad and microcomputer. To examine mesenchymal cell shape, a form factor was calculated for each cell, $\mathrm{FF}=4 \cdot \pi \cdot$ Area/Perimeter ${ }^{2}$. Using this formula, a circle would have a form factor of 1 ; as the cell becomes more elongate, the factor approaches zero. Data were analyzed using $t$-test; a probability of less than or equal to 0.05 was considered significant.

\section{RESULTS}

\section{Incidence and Types of Anomalies}

The incidence of resorptions and anomalies in these embryos has been described previously [37]. Briefly, on the 10th day of development, Snell embryos (33\%) exhibited neural tube closure defects of the cervical $(78 \%)$ or spinal (22\%) neural tube. None of the CFLP or CD-1 embryos exhibited a defect of neural tube closure on either the 10th or 11 th days. By the 11th day, a similar (33\%) incidence of neural tube closure defects was observed in the Snell embryos with an increase in the proportion of anomalies found in the cephalic region ( $83 \%$ ) compared with the spinal neural tube $(17 \%)$. Figure 1.A illustrates the normal appearance of a CFLP control embryo and Fig. 1.B-D the two types of cephalic defects observed in Snell embryos. Figure 1.B illus- trates an apparent deficit in midbrain development. In this particular embryo, the midbrain is considerably reduced in size and the surface ectoderm only partially covers the neuroepithelium. In Fig. 1.C, the neural tube has failed to close in the entire cephalic region and the hypertrophied neuroepithelium has everted over the surface ectoderm. Despite the fact that it is exposed, the hindbrain appears to be well developed (Fig. 1.D).

\section{Histological Observations}

The neuroepithelium of control embryos exhibited a typical pseudostratified configuration, a highly developed luminal surface and smooth lower surface. Interphase nuclei were located near the base of the cells, while dividing cells were seen at the lumen. At all stages examined, the neural tube was closed throughout the cephalic region.

Mesenchymal cells had a stellate appearance and were loosely packed between surface ectoderm and neuroepithelium. They contacted both other mesenchymal cells as well as neuroepithelium and surface ectoderm. Neural crest cells were observed to exit from the neuroepithelium and enter the mesenchyme, particularly in regions near the developing branchial arches. Blood vessels had not yet begun to colonize the neuroepithelium, although paired dorsal aortae were observed in the mesenchyme parallel to the neuroepithelium.

Overall, there was little cell death in either the neuroepithelium or mesenchyme. Figure 2 illustrates the typical cross-sectional appearance of the cephalic region in a CFLP control embryo.

The histological appearance of Snell embryos was very different. The neural tube had often failed to close in midbrain and hindbrain regions, and in some cases, the neuroepithelium was everted over the surface ectoderm-a histological picture similar to that observed using SEM (Fig. 1.C,D). Figure 3 illustrates the appearance of one such embryo. Neuroepithelial hypertrophy was especially evident in the midbrain (Fig. 3.A). Neuroepithelial cells were everted over the surface ectoderm (Fig. 3.B), and the neuroepithelium was very infolded (Fig. 3.B,C). At deeper levels, secondary lumina formed (Figs. 3.C,D, 4.B) and rather than maintaining a smooth basal surface, neuroepithelial cells penetrated the mesenchymal compartment (Figs. 3.C, 4.C). At higher magnification, the neuroepithelial stratification around secondary lumina (Fig. 4.B), early vascularization (Fig. 4,B,C) and presence of mitotic cells deep within the neuroepithelium (Fig. 4.C) can be seen.

In other Snell embryos, a very different histological picture was observed. Rather than the apparent hypertrophy, eversion and infolding of the neuroepithelium, in approximately $48 \%$ of the embryos with neural tube defects, there was a reduction in thickness of the neuroepithelium. There was considerable cell death in the neuroepithelium of these embryos, primarily in the midbrain region. In addition, neural crest cells-near their origin in the neuroepithelium and also near their final destination in the branchial arches-were also pyknotic (Fig. 4.A). There was little generalized mesenchymal or surface ectodermal death, however.

In both cases, mesenchymal cells appeared to be very attenuated with highly elongated, filiform processes which appeared rarely to contact other mesenchymal cells. There also appeared to be a slight reduction in the overall number of mesenchymal cells compared with controls. 


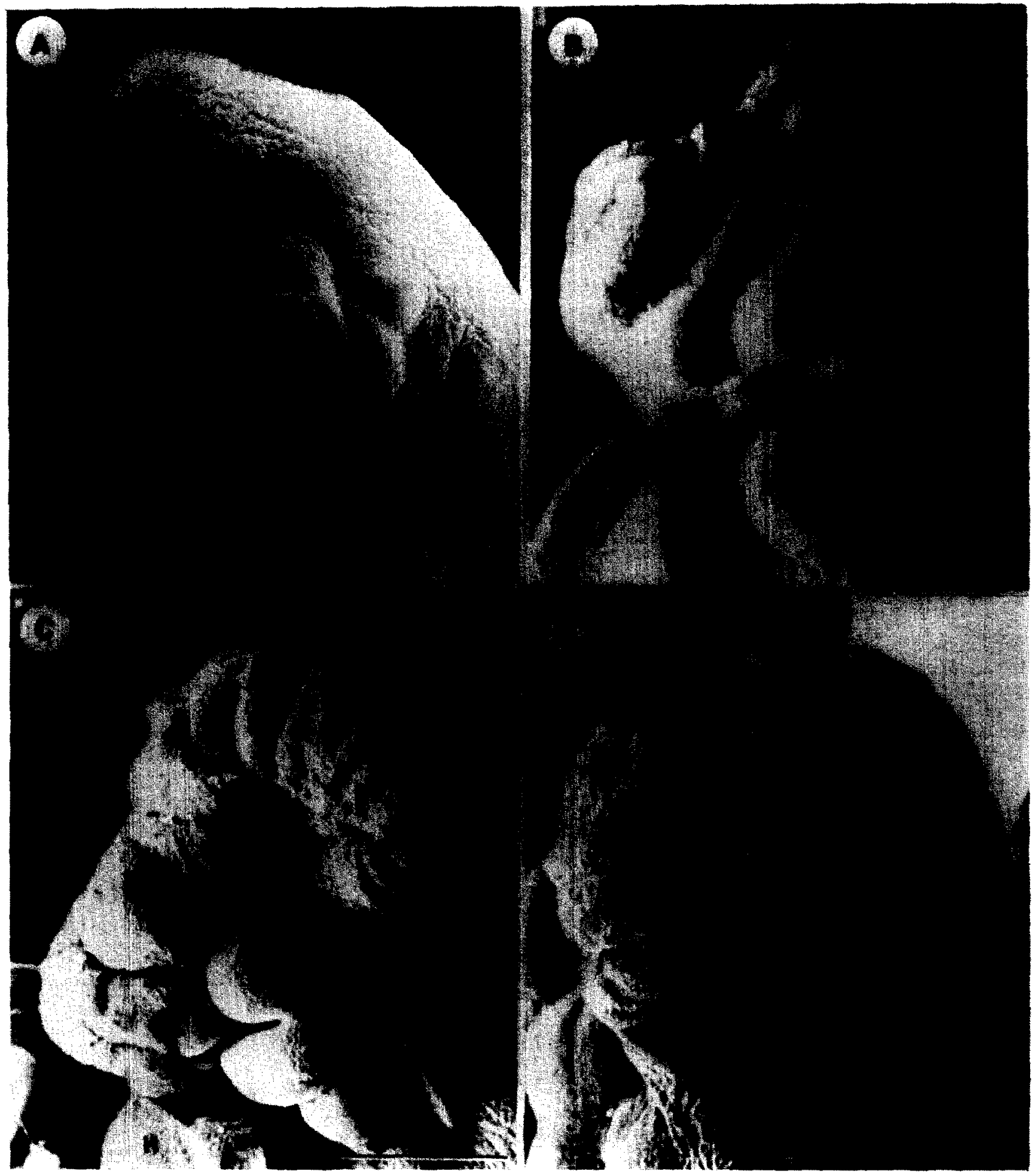

FIG. 1. Scanning electron microscope photomicrographs of a CFLP control (A), and two Snell embryos (B-D). Scale bars $=500$ micrometers. $H=$ heart. $A$. Side view of the cephalic region of a control embryo. Note that the neural tube is closed throughout its cephalic extent; note also the well formed facial region. B. This Snell embryo illustrates the typical appearance of the second reduction anomaly which is especially evident in the midbrain region (arrowed). C,D. Side and dorsal views of a Snell embryo illustrating neuroepithelial hypertrophy and eversion which is particularly evident in C. Arrows indicate regions in which the neuroepithelium has folded over the surface ectoderm. The hindbrain appears well developed, although uncovered (D). The spinal neural tube of this embryo was closed, but was very kinked; a ' $Z$ ' shaped ridge was observed along its dorsal surface. 

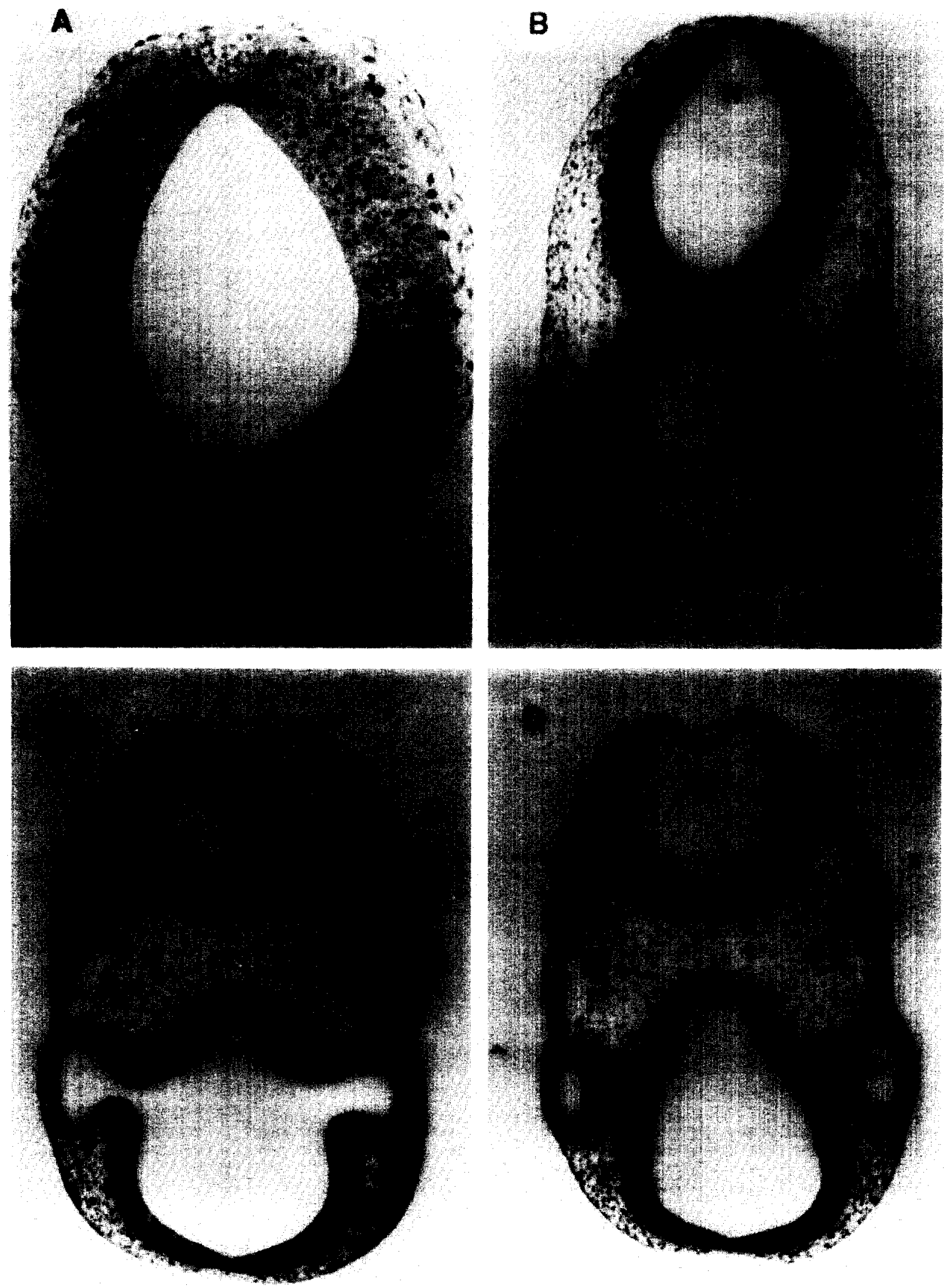

FIG. 2. Transverse sections through the cephalic region of a control embryo encompassing midbrain (A), upper forebrain and hindbrain (B), level of invaginating optic vesicles (C), and fourth ventricle and optic vesicles (D). Note the tight packing and organized appearance of the neuroepithelium and cellularity of the mesenchyme. $\mathrm{H}=$ hindbrain, $\mathrm{OV}=$ optic vesicle. Scale bars $=\mathbf{2 0 0}$ micrometers. 

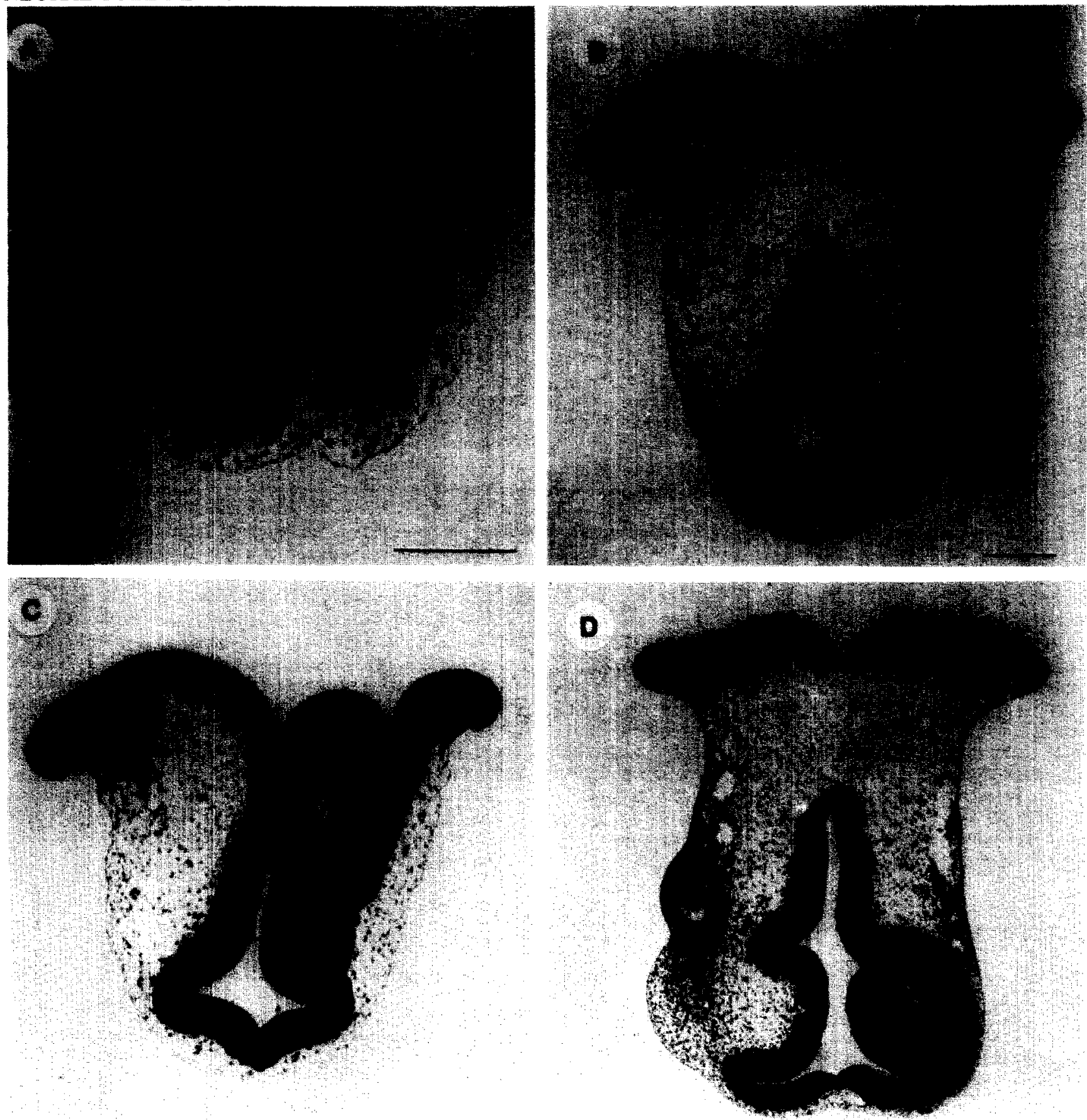

FIG. 3. Transverse sections through the cephalic region of a Snell embryo illustrating a condition similar to that seen in Fig. 1.C,D. There is considerable neuroepithelial hypertrophy at all levels, especially prominent in the midbrain (A). Eversion and infolding of hindbrain neuroepithelium $(B, C)$, as well as slight asymmetrical development of the forebrain $(B)$ can be seen at sequential levels. More caudally, the hypertrophy has resulted in neuroepithelial stratification, formation of a secondary lumen $(L)$, and herniation of neuroepithelial cells into the mesenchymal space (arrowed). At the region of the developing optic vesicles (D), development is again asymmetrical, with one well formed eye rudiment and a second area of stratification and secondary lumen formation. Note that at all levels the forebrain has closed and the hindbrain remains open. Scale bars $=\mathbf{2 0 0}$ micrometers.

\section{Ultrastructural Observations}

Ultrastructurally, the cranial neuroepithelium of control embyros appeared well organized, with wedge-shaped cells contacting both lumen and basal lamina. The lumen typically contained portions of extruded cytoplasm above thick bundles of microfilaments which inserted into apical junctional complexes. Interphase nuclei were located in the basal onethird of the cells while metaphase plates were seen only near the luminal surface. Microtubules were present as were Golgi apparatus, mitochondria and scattered ribosome rosettes. Figure 5.A illustrates the typical appearance of the neuroepithelium in the hindbrain of a CFLP control.

The smooth basal surface of the neuroepithelium (Fig. 
5.C) was bounded by a well-developed basal lamina consisting of a lucid layer, punctate dense layer and outer region with collagen fibrils. A number of blood vessels approached, but did not yet contact the basal lamina, and only a few mesenchymal cell processes approached the neuroepithelial basal lamina at this stage.

Mesenchymal cells had many attenuated processes which typically contacted surface ectoderm and neuroepithelium as well as other mesenchymal cells. Contacts of the gap junctional variety were most commonly observed between these cells. Cell-cell contact was particularly apparent in the neural crest pathway. The dense mesenchymal cytoplasm was filled with ribosomes, cytoskeletal elements, mitochondria and pinocytotic vesicles. Figure 6.A illustrates a typical field of control mesenchymal cells. There was very little cell death in either neuroepithelium or mesenchyme, although occasionally pyknotic cells were found within the neuroepithelium just below the region of emergence of the neural crest.

Ultrastructurally, two different patterns of anomalies were observed in Snell embryos. The first was typical of the histological picture of neuroepithelial hypertrophy described above. In this case the neuroepithelium, rather than maintaining a typical pseudostratified appearance, had become stratified, often appearing to organize around a secondary lumen. Metaphase plates were buried within the neuroepithelium rather than at the luminal surface. Often, the stratified neuroepithelial cells had both an apical and a basal cell process, giving the region a loosely woven appearance (Fig. 5.B).

The luminal surface was also atypical. The cells were often rounded, bulging into the lumen and were attached to each other, not by the typical junctional complex with its normal complement of microfilaments, but well below the luminal surface by what appeared to be very small occluding junctions. Areas of cell bulging and atypical junctional development and positioning were bounded by regions typical of control embryos, giving the surface a wavy or slightly scal- loped appearance. This anomalous pattern was not localized to a single brain region, but was observed throughout the cephalic region in these embryos.

The neuroepithelial basal lamina in these embryos appeared similar to that seen in control embryos, but mesenchymal cells appeared to have considerably longer processes and much less cytoplasm than in controls. It also appeared that there were fewer mesenchymal cells per field and they made fewer mesenchymal-mesenchymal contacts than control mesenchyme (Fig. 6.B).

In the other condition, neuroepithelial cells were very reduced in size and often appeared slightly shrunken. There was an increase in intercellular space and emphasis on apical junctions-which appeared otherwise normal. There was considerable cell death in the wall of the neural tube, which was confined primarily to the midbrain region in all embryos examined.

The basal surface was often wavy, and the basal lamina incomplete; with collagen fibrils less prominent than in controls. Endothelial cells had also begun to invade the neuroepithelium, their hook-like projections often penetrating the spaces between neuroepithelial cells. Figure 5.D illustrates premature endothelial contact and resulting neuroepithelial disorganization in the midbrain region. Many vessels appeared to contain, in addition to nucleated red blood cells, large monocyte-macrophage like cells. Although presumably of phagocytic capacity, the appearance of these cells did not always strictly correspond to areas of greatest cell death, but were found in normal position for later blood vessel colonization of the nervous system.

Like the first condition, mesenchymal cells appeared to have a reduced cytoplasmic volume; either with very little cytoplasm and rounded profile or with little cytoplasm and much longer than normal cell processes.

There was considerable neural crest mesenchyme cell death near the neural tube, along their migratory route and in the branchial arches, while other mesenchymal cells were almost uniformly spared.

\section{FACING AND FOLLOWING PAGES}

FIG. 4. A. Close up of the neuroepithelium and surrounding mesenchyme in an embryo with reduction-type abnormality. Note the neuroepithelial vacuolation along the luminal surface and considerable neuroepithelial and neural crest cell death (arrowed). B. High magnification view of neuroepithelial cell island and forming secondary lumen (L). Note the considerable stratification and premature presence of vessels (arrowed) in this region. C. Higher power view of another region of neuroepithelial stratification in which the neuroepithelial cells, rather than maintaining a smooth basal surface, have begun to penetrate the mesenchyme. Note the presence of vessels and atypical position of mitotic figures (arrowed) deep within the neuroepithelium. Scale bars $=100$ micrometers.

FIG. 5. Transmission EM photomicrographs through the neuroepithelial luminal surface in control (A) and Snell (B) embryos and through the basal portion of the neuroepithelium in control (C) and Snell (D) embryos. Scale bars $=2$ micrometers. A. Typical appearance of the neuroepithelium in the hindbrain of a control embryo. The luminal surface (L) is irregular with cytoplasmic extrusions and well formed junctional complexes. There is little extracellular space and mitotic cells $(M)$ are typically found near the lumen. B. Appearance of the hindbrain neuroepithelium in a Snell embryo with neuroepithelial hypertrophy. The luminal surface appears very irregular, and many cell processes with increased extracellular space separate the luminal surface and the mitotic cells $(\mathbf{M})$. C. Basal region of the neuroepithelium of a control embryo illustrating the typical nuclear placement and tightly packed cells. The basal lamina (brackets) separates the neuroepithelium from the subjacent mesenchymal space (mes). Mesenchymal cell processes (arrowed) approach and contact the basal lamina. D. Basal surface of the neuroepithelium in the midbrain region of a Snell embryo with the second, reduction-type condition. There is premature endothelial (E) contact of the neuroepithelial basal lamina (brackets) producing severe disorganization of the lower portion of the neuroepithelium. Note the considerable increase in extracellular space and numerous neuroepithelial cell processes in this region.

FIG. 6. Typical fields of mesenchymal cells from the hindbrain region of a CD-1 control $(A)$ and from a Snell embryo (B). Scale bars $=2$ micrometers. A. Control mesenchymal cells exhibit their normal stellate appearance, typical packing density and numerous cell-cell contacts. They have more numerous, shorter processes than mesenchyme in Snell embryos. B. Mesenchyme cells from a similar region in a Snell embryo illustrating their highly elongate form, lack of cytoplasm and few cell-cell contacts. 


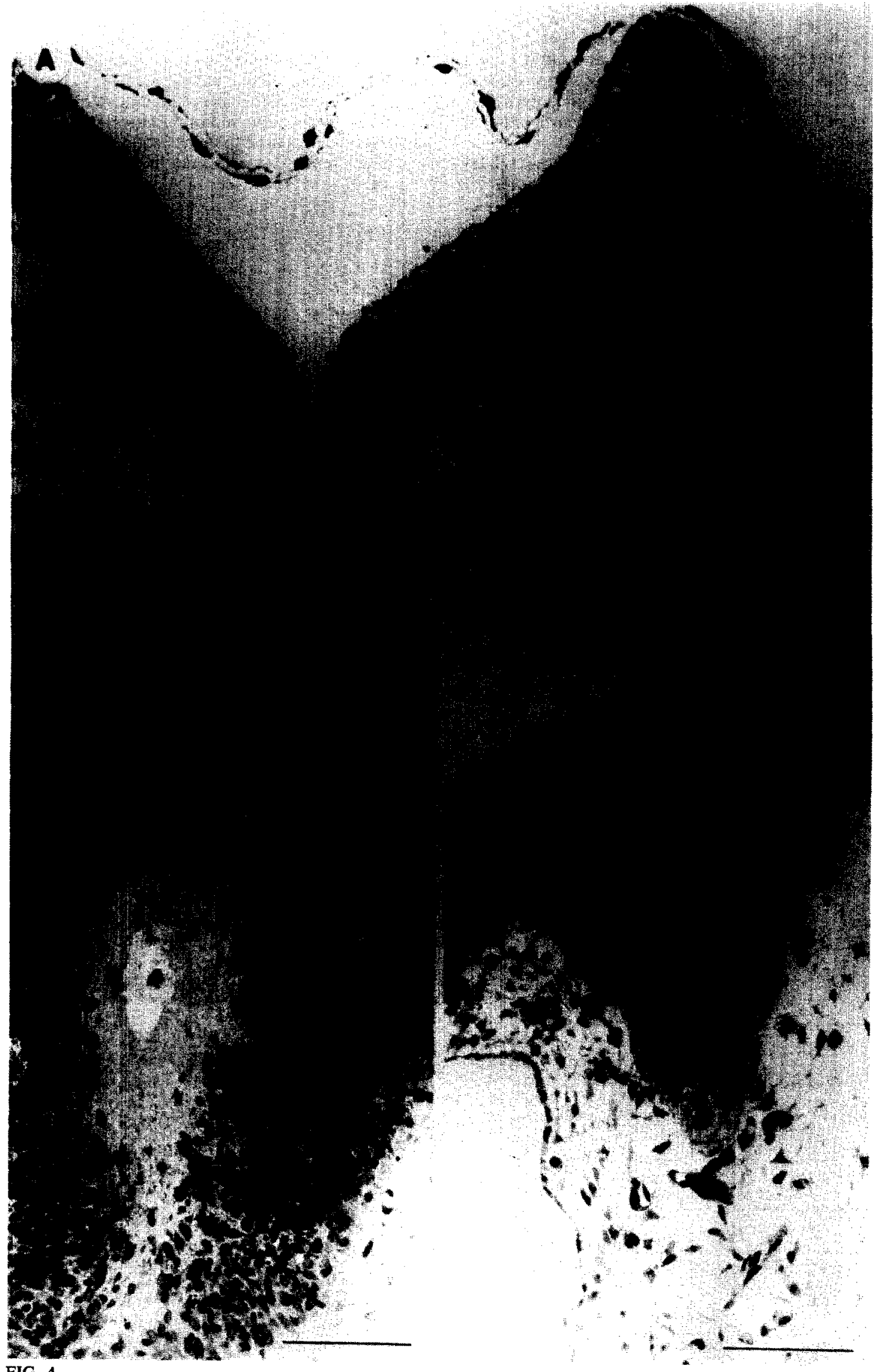


O'SHEA

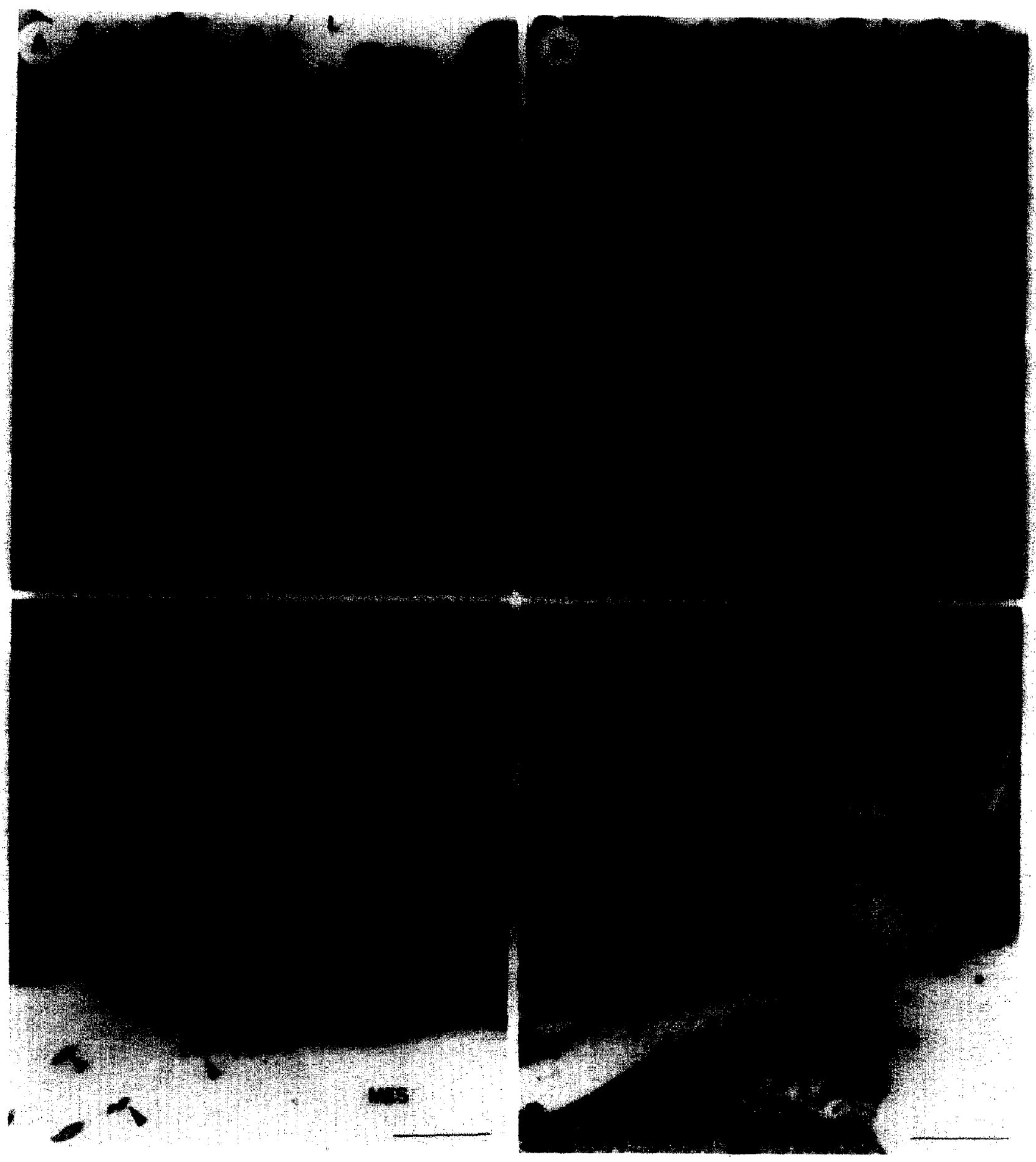

FIG. 5. 

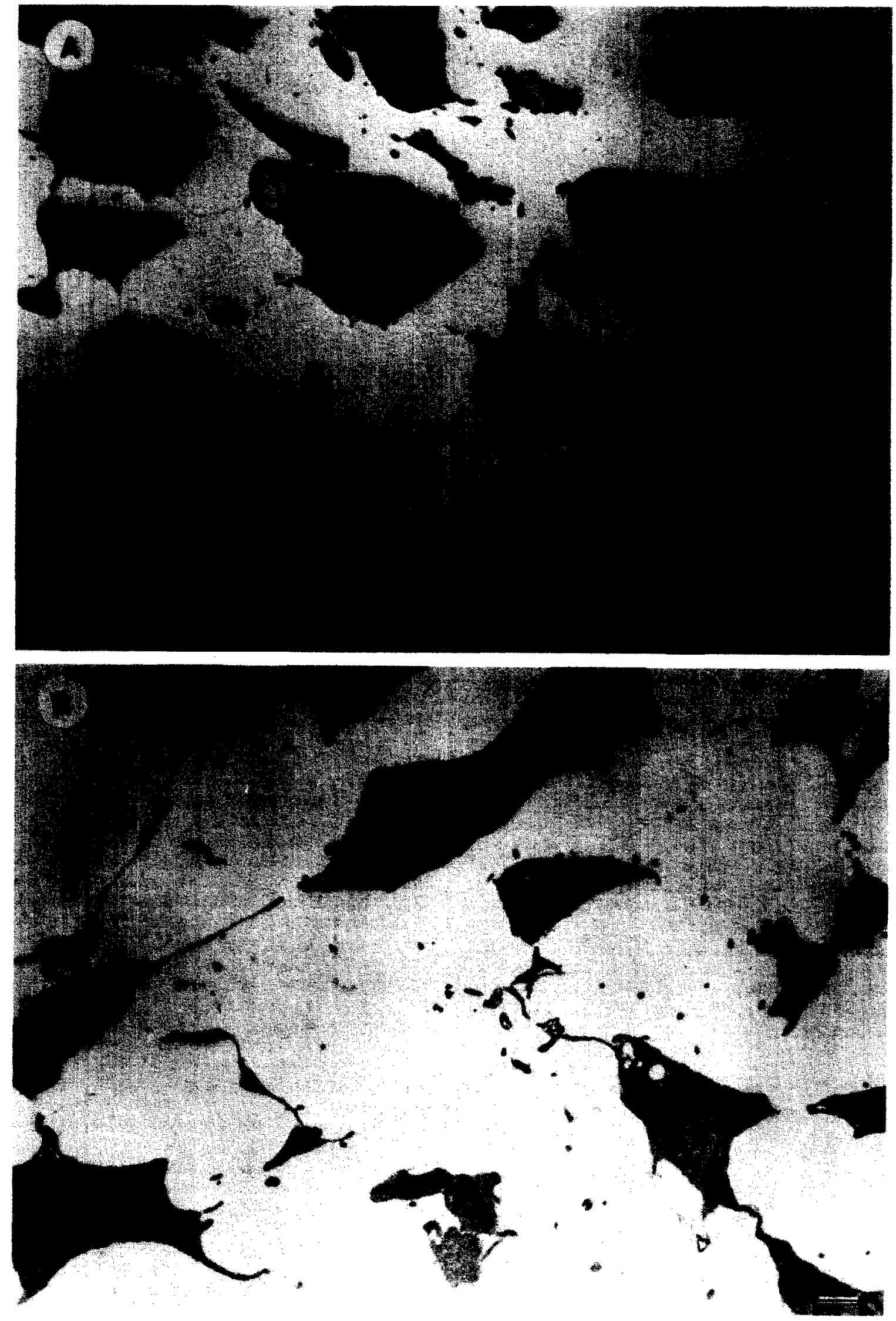

FIG. 6. 
TABLE 1

\begin{tabular}{lccccc}
\hline Group & $\begin{array}{c}\mathrm{n} \\
\text { Cells }\end{array}$ & Area* & Perimeter & $\begin{array}{c}\text { Greatest } \\
\text { Length }\end{array}$ & $\begin{array}{c}\text { Form } \\
\text { Factor }\end{array}$ \\
\hline Control & 153 & $50.6 \pm 1.9 \dagger$ & $38.0 \pm 0.8$ & $14.1 \pm 0.4$ & $0.47 \pm 0.014 \ddagger$ \\
Snell & 188 & $45.4 \pm 1.6$ & $39.4 \pm 1.0$ & $14.2 \pm 0.3$ & $0.39 \pm 0.012$ \\
\hline
\end{tabular}

*mean \pm S.E.M.

$\dagger t(339)=2.09, p \leqslant 0.05$.

$\ddagger t(337)=4.29, p \leqslant 0.05$.

\section{Morphometric Analysis}

When mesenchymal cell parameters were analyzed, the number of contacts per cell was significantly reduced in Snell mesenchyme $(0.22 \pm 0.04)$ compared with $\mathrm{CD}-1$ controls $(0.66 \pm 0.03) ; t(48)=8.72, p \leqslant 0.05$. In addition, mesenchymal cell area was significantly decreased in Snells (45.4 micrometers $^{2} \pm 1.6$ ) compared with controls (50.6 micrometers ${ }^{2} \pm 1.9$ ). There was no significant difference in cell perimeter or length, but when the form factor was calculated as an indicator of cell shape, Snell mesenchyme cells were significantly more elongate $(F F=0.39 \pm 0.012)$ than controls $(F F=0.47 \pm 0.014)$. Table 1 summarizes these data.

\section{DISCUSSION}

Previous reports on the development of these embryos have also identified two types of neural tube defects; hypertrophy, or so-called "overgrowth" of the CNS similar to our first condition, and the second "clefting" of the hindbrain and midbrain [52]. Neuroepithelial hypertrophy has been suggested to both produce [40] and to result from $[15,45]$ closure defects of the neural tube. Similarly, both accelerated [11] and delayed [44] neuroepithelial development relative to mesenchymal growth has been suggested to produce defects of neural tube closure; and lack of developmental synchrony between mesenchymal and epithelial structures has repeatedly been invoked as a causative factor in many developmental anomalies $[4,32]$.

In the first case, neuroepithelial hypertrophy was accompanied by stratification and formation of cell islands and secondary lumina within the neuroepithelium. Neuroepithelial tissue was highly infolded and everted over the surface ectoderm in these embryos.

In the second condition, there was marked loss of neuroepithelial cytoplasm and significant neuroepithelial and neural crest cell death. The neuroepithelial basal lamina was often incomplete and patchy and endothelial cell processes made premature contact and entered the neuroepithelium at regions of basal lamina rarefaction. It is unclear at this time if the neuroepithelial cells did not produce sufficient basal lamina components to exclude premature vascular ingrowth or whether endothelial cells actively degraded basal lamina components (cf., [17]), possibly to release phagocytic cells into regions of neuroepithelial damage.

Interestingly, a very similar pattern has been described in $T / T$ embryos. There is, in addition to increased neuroepithelial intercellular space, a depletion of the basement membrane in regions of premature mesenchymal/neuroepithelial contact, and herniation of the neuroepithelium into the mesenchymal compartment [53].

Unfortunately, it is not possible to attribute these two types of anomaly to different unbalanced chromosome conditions. Eicher and Washburn [13] reported that at term, fetuses with exencephaly and those which were small and apparently normal were both chromosomally $2,2,4^{2}, 4-i . e .$, there was a duplication of the end of chromosome 2 and a deficiency for the distal end of chromosome 4. Kirk (personal communication) has shown the other unbalanced situation; $2,2^{4}, 4,4$, in retarded but apparently normal fetuses, while she observed the $2,2,4^{2}, 4$ situation only in fetuses with exencephaly. Karyotype analyses in combination with detailed microscopic studies at the early stages of development must be carried out to determine if there is an association between the unbalanced situations and the two types of anomalies observed in the current investigation.

Mesenchymal cell alterations were very similar in both conditions, appearing abnormally elongate with numerous filiform processes, significantly reduced in area and having fewer cell-cell contacts than control mesenchyme.

Basal lamina and mesenchyme have previously been suggested to play an important structural role in neural tube closure; the basal lamina forming a rigid foot-hold along which neuroepithelial cells elongate and become wedgeshaped [22]; and mesenchyme supporting and participating in the formation of the bi-convex shape of the neural folds [30]. A considerable portion of the volume of the headfolds of the early embryo is composed of mesenchyme and its surrounding extracellular matrix. This matrix and the neuroepithelial basal lamina have several similar basic components: glycosaminoglycans which, with the exception of hyaluronate are also covalently linked to protein as proteoglycan; collagen; glycoproteins, fibronectin and laminin; ions and water $[25,26,41,43]$. Extracellular matrix materials are synthesized by neuroepithelium, surface ectoderm, and mesenchyme and are released into the matrix, and retained at the cell surface [26] and basal lamina [24].

Defects of basal lamina and/or mesenchyme, whether gene: loop-tail [12], Splotch [28], delayed Splotch (O'Shea and Rheinheimer, in preparation), Patch [14], $T$ mutant [53]; translocation: Ts 12 (Putz, as cited in [19]); or teratogeninduced: vitamin A [29]; hyaluronidase [1,49]; tunicamycin [35]; B-D-xyloside [31]; cis-hydroxyproline [36]; have been associated with the development of neural tube closure defects.

Mesenchymal abnormalities appear to underlie the formation of neural tube defects in other neurological mutants as well. Waterman [57] has described a reduction in size of 
the anterior neural folds in open eyelid (oel) mice and striking blebbing and abnormal shaping of mesenchymal cells during the period of neural fold elevation. Similarly, $t^{9} / t^{9}$ embryos are characterized by fewer and blunted mesenchymal cell processes, which also have been reported to make few cell-cell junctions [53].

Copp and Wilson [12] have described a reduction in the proportion of non-sulfated glycosaminoglycans (hyaluronate) to sulfated glycosaminoglycans (chondroitin sulfate) in the extracellular matrix, on mesenchymal cell surfaces and along the neuroepithelial basal lamina in loop-tail embryos, and suggest that changes in the type of glycosaminoglycan present might interfere with cell migration and/or proliferation, producing inadequate mesenchymal support for the neural folds.

Since there is abundant evidence that cell surface molecules may control cytoskeletal organization [50], an alteration in the type, relative proportion, or time of appearance of cell surface/extracellular matrix components might explain the abnormally elongate form of the mesenchymal cells. In addition to determining cell shape [7], extracellular macromolecules play an important role in controlling cell adhesion [18], cell migrations [5, 6, 8, 33, 43], as well as the nature and positioning of the intercellular junctions $[34,38]$.
Similarly, because in their hydrated form, extracellular matrix components such as hyaluronate may be involved in maintaining extracellular spaces and in inhibiting premature contact between tissue types [55], premature endothelial ingrowth into the neuroepithelium may result from alterations in the characteristics of the basal lamina or the extracellular matrix. Recently, Wilson [58] has described an abnormal pattern of vasculogenesis in the telencephalon of loop-tail embryos which have an abnormal glycosaminoglycan distribution at earlier embryonic stages.

An alternative explanation for the reduced number of cell-cell contacts and abnormal elongate appearance of the mesenchymal cells is that they require cell-cell contact to maintain a coordinated reticulum, due to the overall paucity of mesenchymal cells establishment of contact is attempted over large distances. Detailed examination of the nature and time of appearance of extracellular matrix elements should help to clarify these issues.

\section{ACKNOWLEDGEMENTS}

The author is grateful to Ms. J. Rheinheimer, Drs. M. H. Kaufman and J. M. O'Shea for advice and assistance in this study. Supported by NIH Grant NS-21108.

\section{REFERENCES}

1. Anderson, C. B. and S. Meier. Effect of hyaluronidase treatment on the distribution of cranial neural crest cells in the chick embryo. J Exp Zool 221: 329-339, 1982.

2. Arias-Bernal, L. and $H$. W. Jones, Jr. An anencephalic male with XX sex chromosome complement. Am J Obstet Gynecol 99: 877-878, 1967.

3. Bader, P. I., S. M. Haney, R. A. Munsick, S. R. Schubert and M. E. Hodes. Neural tube defects in dup(11q). Am J Med Genet 19: 5-8, 1984.

4. Bersu, E. T. Disruptions in the normal progression of phenotypic development caused by $\mathrm{Ts} 19$ in the mouse. Teratology 19: 19A-20A, 1979.

5. Bolender, D. L., W. G. Seliger and R. R. Markwald. A histochemical analysis of polyanionic compounds found in the extracellular matrix encountered by migrating cephalic neural crest cells. Anat Rec 196: 401-412, 1980.

6. Bolender, D. L., W. G. Seliger, R. R. Markwald and P. R. Brauer. Structural analysis of extracellular matrix prior to the migration of cephalic neural crest cells. Scan Electron Microsc II: $285-296,1981$.

7. Bornstein, P. and J. F. Ash. Cell surface-associated structural proteins in connective tissue cells. Proc Natl Acad Sci USA 74: 2480-2484, 1977.

8. Boucaut, J.-C., T. Darribere, T. J. Poole, H. Aoyama, K. M. Yamada and J.-P. Thiery. Biologically active synthetic peptides as probes of embryonic development: a competitive peptide inhibitor of fibronectin function inhibits gastrulation in amphibian embryos and neural crest cell migration in avian embryos. $J$ Cell Biol 99: 1822-1830, 1984.

9. Carr, D. H. Chromosomes and abortion. In: Advances in Human Genetics, vol 2, edited by $\mathrm{H}$. Harris and K. Hirschhorn. New York: Plenum Press, 1971, p. 223.

10. Carter, C. O. Multifactorial inheritance revisited. In: Congenital Malformations, edited by F. C. Fraser and V. McKusick. New York: Excerpta Medica, 1970, pp. 227-232.

11. Copp, A. J., M. J. Seller and P. E. Polani. Neural tube development in mutant (curly-tail) and normal mouse embryos: the timing of posterior neuropore closure in vivo and in vitro. $J$ Embryol Exp Morphol 69: 151-167, 1982.
12. Copp, S. N. and D. B. Wilson. Cranial glycosaminoglycans in early embryos of the loop-tail $(L p)$ mutant mouse. $J$ Craniofac Genet Dev Biol 1: 253-260, 1981.

13. Eicher, E. and L. Washburn, Jackson laboratory news. Mouse Newsletter N46: 43, 1977.

14. Erickson, C. A. and J. A. Weston. An SEM analysis of neural crest migration in the mouse. J Embryol Exp Morphol 74: 97$118,1983$.

15. Fowler, I. Responses of the chick neural tube in mechanically produced spina bifida. $J$ Exp Zool 123: 115-151, 1953.

16. Frezal, J., J. Kelley, M. L. Guillemot and M. Lamy, Anencephaly in France. Am J Hum Genet 16: 336-350, 1964.

17. Glaser, B. M., T. Kalebic, S. Garbisa, T. B. Connor, Jr. and L. A. Liotta. Degradation of basement membrane components by vascular endothelial cells: role in neovascularization. In: Development of the Vascular System, edited by J. Nugent and $\mathbf{M}$. $O^{\prime}$ Connor. London: Pitman Medical Publishers, CIBA Foundation Symposium 100: 150-162, 1983.

18. Greenberg, J. H., S. Seppa, H. Seppa, and A. T. Hewitt. Role of collagen and fibronectin in neural crest cell adhesion and migration. Dev Biol 87: 259-266, 1981.

19. Gropp, A. Value of an animal model for trisomy. Virchows Arch [A] 395: 117-131, 1982.

20. Hamerton, J. L. Human Cytogenetics, Vol 1. New York: Academic Press, 1971.

21. Harnden, D. G., J. H. Briggs and J. S. S. Stewart. Nuclear chromatin of anencephalic foetuses. Lancet it: 126-127, 1959.

22. Hay, E. D. Organization and fine structure of epithelium and mesenchyme in the developing chick embryo. In: Epithelial and Mesenchymal Interactions, edited by R. Fleischmajer and R. E. Billingham. Baltimore: Williams and Wilkins, 1968, pp. 31-55.

23. Holman, G. D., B. Erkman, D. L. Zacharias and H. F. Koch. The 18-trisomy syndrome-two new clinical variants. New Engl J Med 268: 982-988, 1963.

24. Kefalides, N. A., R. Alper and C. C. Clark. Biochemistry and metabolism of basement membranes. Int Rev Cytol 61: 167-228, 1979.

25. Kvist, T. N. and C. J. Finnegan. The distribution of GAGs in the axial region of the developing chick embryo. I. Histochemical analysis. $J$ Exp Zool 175: 221-240, 1970. 
26. Manasek, F. J. and A. M. Cohen. Anionic glycopeptides and glycosaminoglycans synthesized by embryonic neural tube and neural crest. Proc Natl Acad Sci USA 74: 1057-1061, 1977.

27. Masterson, J. G. Empiric risk, genetic counseling and preventive measures in anencephaly. Acta Genet 12: 219-229, 1962

28. Morris, G. L. and K. S. O'Shea. Anomalies of neuroepithelial cell associations in the Splotch mutant embryo. Dev Brain Res 9: 408-410, 1983.

29. Morriss, G. M. The ultrastructural effects of excess maternal vitamin A on the primitive streak stage rat embryo. $J$ Embryol Exp Morphol 30: 219-242, 1973.

30. Morriss, G. M. and M. Solursh. Regional differences in mesenchymal cell morphology and glycosaminoglycans in early neural-fold staged rat embryos. J Embryol Exp Morphol 46: $37-52,1978$.

31. Morriss-Kay, G. M. and B. Crutch. Culture of rat embryos with B-D-xyloside: evidence of a role for proteoglycans in neurulation. J Anat 134: 491-506, 1982.

32. Myrianthopoulos, N. C. Congenital malformations: the contribution of twin studies. Birth Defects 14: 151-165, 1978

33. Newgreen, D. and J.-P. Thiery. Fibronectin in early avian embryos: synthesis and distribution along the migration pathways of neural crest cells. Cell Tissue Res 211: 269-291, 1980.

34. Norton, E. K. and C. S. lzzard. The role of fibronectin in the formation of close and focal contacts. J Cell Biol 91: 110A, 1981 .

35. O'Shea, K. S. Tunicamycin-induced defects in the mouse embryo: an in vitro study. In: The Extracellular Matrix, edited by S. P. Hawkes and J. L. Wang. New York: Academic Press, 1982, pp. 147-151.

36. O'Shea, K. S. Effects of cis-hydroxyproline on the developing neuroepithelial basal lamina: a correlated slice culture and whole embryo culturc study. Soc Neurosci Abstr 10: 45, 1984.

37. O'Shea, K. S. and M. H. Kaufman. Chromosome translocation $(\mathrm{T}(2 ; 4) 1 \mathrm{Sn}$ )-induced neural tube defects in the mouse embryo. $J$ Neurogenet 1: 29-38, 1983.

38. Overton, J. Formation of junctions and cell sorting in aggregates of chick and mouse cells. Dev Biol 55: 103-116, 1977.

39. Passarge, E., C. W. True, W. T. Sueoka, N. R. Baumgartner and $K$. R. Keer. Malformations of the central nervous system in trisomy 18 syndrome. J Pediatr 69: 771-778, 1966.

40. Patten, B. M. Overgrowth of the neural tube in young human embryos. Anat Rec 113: 381-393, 1952.

41. Pintar, J. E. Distribution and synthesis of GAG during quail crest morphogenesis. Dev Biol 67: 444-464, 1978.

42. Pitt, D., M. Leversha, C. Sinfield, P. Campbell, R. Anderson, D. Bryan and J. Rogers. Tetraploidy in a liveborn infant with spina bifida and other anomalies. $J$ Med Genet 18: 309-311, 1981.

43. Pratt, R. M., M. A. Larsen and M. C. Johnston. Migration of cranial neural crest cells in a cell-free hyaluronate-rich matrix. Dev Biol 44: 298-305, 1975.
44. Putz, B. and G. Morriss-Kay. Abnormal neural fold development in trisomy 12 and trisomy 14 mouse embryos. I. Scanning electron microscopy. J Embryol Exp Morphol 66: 141-158, 1981.

45. Rokos, J. and J. Knowles. An experimental contribution to the pathogenesis of spina bifida. $J$ Pathol 118: 21-24, 1976.

46. Rott, H.-D., G. Schwanitz, K.-P. Grosse and G. Alexandrow. C $11 \mathrm{D}$ 13-translocation in four generations. Humangenetik 14: 300-305, 1972.

47. Russell, W. L. Splotch: a new mutation in the house mouse. Genetics 32: 102, 1947.

48. Schmid, W., J. P. Muhlethaler, J. Briner and H. Knechtli. Ring chromosome 13 in a polymalformed anencephalic. Humangenetik 27: 63-66, 1975.

49. Schoenwolf, G. C. and M. Fisher. The effects of streptomyces hyaluronidase on neurulating chick embryos. I Embryol Exp Morphol 73: 1-15, 1983.

50. Singer, I. I. The fibronexus: a transmembrane association of fibronectin-containing fibers and bundles of $5 \mathrm{~nm}$ microfilaments in hamster and human fibroblasts. Cell 16: 675-685, 1979.

51. Snell, G. D. X-ray sterility in the male house mouse. J Exp Zool 65: 421-441, 1933.

52. Snell, G. D., E. Bodemann and W. Hollander. A translocation in the house mouse and its effect on development. J Exp Zool 67: 93-104, 1935.

53. Spiegelman, M. Electron microscopy of cell associations in T-locus mutants. In: Embryogenesis in Mammals, edited by $\mathrm{K}$. Elliot and M. O'Connor. Amsterdam: Elsevier. CIBA Foundation Symposium 40: 199-226, 1976.

54. Stein, K. F. and I. A. Rudin. Development of mice homozygous for the gene for loop-tail. J Hered 44: 59-69, 1953.

55. Toole, B. P. Morphogenetic role of glycosaminoglycans (acid mucopolysaccharides) in brain and other tissues. In: Neuronal Recognition, edited by S. H. Barondes. New York: Plenum, 1976, pp. 275-329.

56. Warkany, J., E. Passarge and L. B. Smith. Congenital malformation in autosomal trisomy syndromes. Am J Dis Child 112: $502-517,1966$.

57. Waterman, R. E. Scanning electron microscope studies of central nervous system development. Birth Defects 15: 55-77, 1979.

58. Wilson, D. B. Cerebrovascular pathogenesis in the telencephalon of the loop-tail mouse: a transmission electron-microscopic study. Acta Neuropathol 58: 177-182, 1982.

59. Wright, Y. M., W. E. Clark and W. R. Breg. Craniorachischisis in a partially trisomic 11 fetus in a family with reproductive failure and a reciprocal translocation, $5(6 \mathrm{p}+; 11 \mathrm{q}-) . J \mathrm{Med}$ Genet 11: 69-75, 1974. 